\title{
Avaliação do Comportamento de Risco para Transtornos Alimentares e Distúrbios de Imagem Corporal em Estudantes Universitárias
}

\author{
Risk Behavior Evaluation for Eating Disorders and Body Image Disorders in \\ University Students
}

\author{
Michelle Cristine Nascimento de Melo ${ }^{1}$ \\ Sara Ferreira de Oliveira ${ }^{1}$ \\ Patrícia Vasconcelos Leitão Moreira² \\ Rafaela Lira Formiga Cavalcanti de Lima $^{3}$
}

\section{RESUMO}

Objetivo: Avaliar a prevalência do comportamento de risco para transtornos alimentares e distúrbios de imagem corporal em estudantes universitárias do Curso de Nutrição da UFPB, bem como descrever o perfil socioeconômico e os hábitos gerais de vida das estudantes, verificando as possíveis associações entre essas variáveis e averiguar a associação entre os comportamentos de risco para transtornos alimentares e os distúrbios de imagem corporal entre as universitárias. Metodologia: Trata-se de um estudo tipo transversal. Foram utilizados os instrumentos Eating Attitudes Test (EAT-26) e o Body Shape Questionnaire (BSQ). Os dados foram analisados com o auxílio do programa SPSS, adotando-se o nível de significância de $5 \%(p<0,05)$. Participaram do estudo 99 estudantes, com média e desvio padrão da idade, altura, peso e IMC, sendo $21,5 \pm 4,53$ anos, $1,62 \pm 0,69$ metros, $58,5 \pm 11,7 \mathrm{~kg}$ e IMC $22,1 \pm 4,01 \mathrm{~kg} / \mathrm{m}^{2}$, respectivamente. Resultados: Constatou-se que $27,3 \%$ das estudantes apresentaram risco de desenvolver transtornos alimentares e 76,8\% apresentaram ausência de insatisfação com a imagem corporal, além da associação estatisticamente significativa com a prática de exercício físico $(p=0,011)$, a frequência $(p=0,043)$, o tempo $(p=0,017)$ e a modalidade $(p=0,000)$ com o EAT. Ocorreu associação quanto à insatisfação com a imagem corporal relacionada com a presença de atitudes alimentares de risco para o desenvolvimento de transtornos alimentares $(p=0,009)$. Conclusão: Os resultados desse estudo são bem preocupantes. Verificou-se um grande número de estudantes insatisfeitas com o peso, evidenciando a elevada prevalência do comportamento de risco para transtornos alimentares e a presença de distúrbios da imagem corporal.

\section{DESCRITORES}

Transtornos da Alimentação. Ingestão de Alimentos. Imagem Corporal. Estudantes. Comportamento Alimentar.

\begin{abstract}
Objective: To evaluate the prevalence of risk behavior for eating disorders and body image disorders in college students of the UFPB Nutrition Course, as well as to describe the socioeconomic profile and general life habits of the students, checking the possible associations between these variables and to investigate the association between risk behaviors for eating disorders and body image disorders among college girls. Methodology: This is a cross-sectional study. Eating Attitudes Test (EAT-26) and Body Shape Questionnaire (BSQ) instruments were used. Data were analyzed using the SPSS program, adopting a significance level of $5 \%(p<0.05)$. Ninety-nine students participated in the study, with mean and standard deviation of age, height, weight, and BMI being 21.5 years $\pm 4.53,1.62$ meters \pm 0.69 , $58.5 \mathrm{~kg} \pm 11.7$ and BMI $22,1 \mathrm{~kg} / \mathrm{m}^{2} \pm 4.01$, respectively. Results: It was found that $27.3 \%$ of students were at risk of developing eating disorders and $76.8 \%$ of students had no dissatisfaction with body image in addition to the statistically significant association with physical exercise $(p=0.011)$, frequency $(p=0.043)$, time $(p=0.017)$ and modality $(p=0.000)$ with the EAT. There was an association regarding dissatisfaction with body image related to the presence of eating attitudes at risk for the development of eating disorders $(p=0.009)$. Conclusion: The results of this study are very worrying. There is a large number of dissatisfactions with weight, evidencing the high prevalence of risk behavior for eating disorders and the presence of body image disorders.
\end{abstract}

\section{DESCRIPTORS}

Feeding. Eating Disorders. Body image. Students. Feeding Behavior.

${ }^{1}$ Nutricionista, graduada pela Universidade Federal da Paraiba/UFPB, João Pessoa/PB, Brasil.

Pós-doutorado em Epidemiologia pela Universidade de Liverpool, Professora do Departamento de Nutrição da UFPB, João Pessoa/ PB, Brasil.

${ }^{3}$ Doutorado em Modelos de Decisão e Saúde pela Universidade Federal da Paraíba, Professora do Departamento de Nutrição da UFPB, João Pessoa/PB, Brasil. 
$\mathrm{N}$ as últimas décadas e nos dias atuais o estereótipo ideal de beleza corporal obteve mudanças relevantes, como resultado da forte influência da sociedade moderna que se encontra também em constante evolução. Para ter sucesso e reconhecimento social atualmente, usa-se como referência o corpo humano. Com o passar do tempo ocorreu uma hipervalorização do corpo e, consequentemente, a mídia apropriou-se disso. Negligenciam-se as particularidades individuais, as limitações biológicas e genéticas, ditando-se um padrão de beleza para homens e mulheres ${ }^{1,2}$.

As sociedades, principalmente as ocidentais, vêm apresentando uma preocupação demasiada com os padrões de beleza, além de uma busca incessante pela magreza exagerada ${ }^{3}$. Neste contexto, os transtornos alimentares passam a ser relevantes diante dessa busca exagerada por se inserir nos padrões ditados tanto pela sociedade quanto pela mídia, além dos prejuízos emocionais e sociais ${ }^{4}$.

Os transtornos de comportamento alimentar são denominados como distúrbios psiquiátricos de etiologia multifatorial caracterizados por consumo, padrões e atitudes alimentares extremamente distorcidas e de preocupação excessiva com o peso e a forma corporal ${ }^{5}$.

Os transtornos mais conhecidos são anorexia nervosa e bulimia nervosa, ambos caracterizados pela preocupação excessiva com o peso, principalmente por jovens do sexo feminino. $O$ excesso de peso torna-se um problema para ambos, bem como o ganho de peso. Portanto, tendo como objetivo o emagrecimento, em que a solução ditada é a dieta altamente restritiva ou a indução da expurgação de alimentos consumidos ${ }^{6}$.

A anorexia é compreendida pela resistência do paciente em alcançar e manter um peso adequado para a sua altura, o medo extremo em aumentar o peso corporal e recusa do alimento associada a uma percepção distorcida da sua imagem corporal ${ }^{7}$. A bulimia é caracterizada por um ciclo constituído por dieta restritiva, compulsão alimentar e purgação. O sentimento de culpabilidade também é apresentado após a ingesta de alguma refeição, sendo compensados posteriormente. A restrição tem papel fundamental no início e perpetuação do quadro ${ }^{8}$.

A imagem corporal está relacionada à percepção do tamanho e a forma do nosso corpo e os sentimentos em relação à forma física. O modo em que o indivíduo enxerga seu corpo é predominantemente alterado pela imaginação de que o mesmo não o pertence ${ }^{9}$.

Diante do exposto, tendo em vista a relação entre transtornos alimentares e distúrbios de imagem corporal em mulheres, o presente trabalho apresenta relevância devido ao grau de importância desses transtornos alimentares, bem como, de suas complexidades e interferência na vida dos indivíduos acometidos pelos mesmos e daqueles que convivem com esses.

Desse modo, a presente pesquisa se propôs a avaliar a prevalência do comportamento de risco para transtornos alimentares e de distúrbios de imagem corporal em estudantes universitárias do Curso de Nutrição da UFPB, como também, descrever o perfil socioeconômico e os hábitos gerais de vida das estudantes, verificando as possíveis associações entre essas variáveis e averiguar a 
associação entre os comportamentos de risco para transtornos alimentares e os distúrbios de imagem corporal entre as universitárias.

\section{METODOLOGIA}

Trata-se de um estudo tipo corte transversal. Foram avaliadas as alunas do Curso de Nutrição da Universidade Federal da Paraíba (UFPB) do $1^{\circ}$ ao $6^{\circ}$ período. Os dois últimos semestres do curso não foram incluídos, pois os alunos fazem seus estágios supervisionados e não se encontram no ambiente universitário. O tamanho da amostra foi determinado por cálculos realizados no OpenEpi (versão 3.01), sendo adotada uma frequência de $50 \%$ do evento, $5 \%$ de erro amostral e $90 \%$ de intervalo de confiança. De acordo com esses parâmetros, verificou-se que seria necessário um tamanho amostral total de cem (100) estudantes com base no número total de alunas regularmente matriculadas e ativas no período 2018.2.

A coleta de dados foi realizada por três alunas de graduação do Curso de Nutrição, devidamente treinadas e responsáveis pela pesquisa, sendo realizada durante as aulas de graduação, com permissão antecipada do professor responsável pela disciplina e consistiu na aplicação do instrumento de coleta de dados autopreenchível.

O instrumento utilizado foi a junção de dois questionários validados anteriormente, que avaliam separadamente comportamentos sugestivos à transtornos alimentares ${ }^{10}$, como a anorexia nervosa e a bulimia nervosa, bem como distorção da imagem corporal ${ }^{11}$. Além de um questionário, baseado em informações de outros estudos com questões fechadas acer- ca do perfil demográfico e socioeconômico, hábitos gerais de vida, situação afetiva e a prática de exercício físico (frequência, tempo e modalidade).

O primeiro questionário aplicado foi o Eating Attitudes Test (EAT-26) ou Teste de atitudes alimentares, que apresenta 26 questões. O EAT-26 é um instrumento que foi desenvolvido por Garner e Garfinkel ${ }^{12}$, que indica a presença de padrões alimentares anormais, além de fornecer um índice de gravidade com relação a preocupações típicas de pacientes com transtorno alimentar e intenção de emagrecer e medo de ganhar peso ${ }^{13}$.

Outro questionário utilizado foi o Body Shape Questionnaire (BSQ) ou Questionário de imagem corporal que possui 34 questões ${ }^{14}$. Adaptado e validado para a população brasileira por Di Pietro e Silveira ${ }^{11}$, com o intuito de avaliar a insatisfação com a imagem corporal, tendo em vista a autopercepção nas últimas quatro semanas. Esse questionário por sua vez fornece uma avaliação contínua. Ele avalia tanto o papel do distúrbio em desenvolvimento, a sua manutenção e atua também na resposta ao tratamento de alguns transtornos alimentares como a anorexia e a bulimia.

Os dados foram digitados no Excel e posteriormente transferidos para o programa estatístico de análise de dados, Statistical Package for the Social Sciences (SPSS) versão 22. A análise estatística foi realizada inicialmente pelo teste de normalidade de Kolmogorov-Smirnov das variáveis contínuas, essas mesmas variáveis foram analisadas por medidas de tendência central, sendo a média e mediana, além do desvio-padrão. Para a análise das possíveis associações entre os distúrbios alimentares e o de imagem corporal, 
além das associações entre as prevalências encontradas com as variáveis, foi utilizado o teste Qui-Quadrado de Pearson. Em todo o estudo foram considerados significativos os testes que obtiveram um nível de significância menor que $5 \%$, p-valor $<0,05$.

A análise dos dados do EAT-26 possui escala de Likert, com seis alternativas de resposta, que pontuam de 0 a 3: "sempre" (3 pontos), "muito frequentemente" (2 pontos), "frequentemente" ( 1 ponto), "às vezes" ( 0 ponto), "raramente" (0 ponto), "nunca" (0 ponto). Porém, a questão 25 possui a pontuação invertida, sendo considerada as respostas "sempre", "muito frequentemente" e frequentemente" com a pontuação de 0 ponto e as de respostas "às vezes" (1 ponto), "raramente" (2 pontos) e "nunca" (3 pontos). Os itens são agrupados conforme os seguintes aspectos: Fator 1: Dieta - as questões refletem recusa patológica às comidas de alto teor calórico e preocupações com a forma física; Fator 2: Bulimia nervosa - refletem pensamentos sobre comida e atitudes bulímicas; Fator 3: Controle oral - refletem o autocontrole em relação a comida e reconhecem pressões sociais no ambiente para ganhar peso.

O resultado é considerado indicador de risco para o desenvolvimento de transtorno alimentar, quando o escore formado pelo somatório de respostas positivas for igual ou $>21$, de 10 a 20 baixo risco e de 0 a 9 fora de risco.

Em relação ao questionário $B S Q$, também com escala de Likert, possui seis possibilidades de respostas: "sempre" (6 pontos), "muito frequentemente" (5 pontos), "frequentemente" (4 pontos), "às vezes" ( 3 pontos), "raramente" (2 pontos) e "nunca" (1 ponto). $\mathrm{O}$ resultado do teste BSQ é a somatória dos 34 itens contidos no questionário e sua classificação reflete os níveis de preocupação com a imagem corporal, tais como: Ausência de insatisfação com a imagem corporal (escore menor ou igual a 110); Leve insatisfação com a imagem corporal (110-138 pontos); Moderada insatisfação com a imagem corporal (138-167 pontos); Grave insatisfação com a imagem corporal (>167 pontos). Os itens desse questionário são agrupados conforme os seguintes aspectos: Autopercepção da forma corporal (questões 4, 2, 21, 17, 24, 28, 22, 23, 30, 14, 3, 6, 34, 16, 9, 5, 11, 10, 1, 33, 15 e 19); Percepção Comparativa da imagem corporal (questões 31, 20, 29, 12 e 25); Atitude em relação à alteração da imagem corporal (questões 32, 26, 7, 18, e 13); Alterações Intensas da percepção corporal (questões 8, 27).

Para a realização da pesquisa foi redigido para a ciência e o consentimento dos participantes, um Termo de consentimento livre e esclarecido, respeitando as diretrizes éticas contempladas na Resolução 466/12, do Conselho Nacional da Saúde. O presente trabalho foi escrito observando todos os aspectos éticos e foi submetido para aprovação do Comitê de Ética em Pesquisa do CCS/ UFPB com o Parecer $n^{\circ}$. 2.841.492.

Os dados foram utilizados somente para fins de pesquisa, sendo assim, foi assegurado o sigilo das informações individuais, garantindo aos voluntários da pesquisa completa autonomia, anonimato, privacidade e isenção de qualquer risco individual ou coletivo. O entrevistado foi resguardado também de qualquer constrangimento moral e ético. Todos os indivíduos que aceitaram participar da pesquisa assinaram o Termo de consentimento livre e esclarecido (TCLE). 


\section{RESULTADOS}

Participaram do estudo 99 estudantes do sexo feminino, com idade média de 21,5 anos, altura média de 1,62 metros, peso médio de $58,5 \mathrm{~kg}$, sendo que $68,8 \%$ encontravam-se eutróficas, $16,7 \%$ com excesso de peso e $14,6 \%$ com baixo peso.

Para verificar a satisfação com o peso e calcular o IMC desejado, foi solicitado às estudantes que respondessem o seu peso desejado e a altura desejada. Quanto a satisfação com o peso, 51,7\% demonstraram o desejo de perder peso e $32,6 \%$ ganhar peso, as demais se encontravam satisfeitas com o seu peso corporal atual.

Constatou-se que $27,3 \%$ das estudantes apresentaram um escore $\geq 21$, que representa um comportamento de risco para desenvolvimento de transtornos alimentares. Quanto ao BSQ, 76,8\% das estudantes não apresentaram alguma distorção da imagem corporal, porém $23,2 \%$ dessas estudantes apresentaram algum grau de distorção de sua percepção corporal.

Ocorreu associação estatisticamente significativa entre a renda familiar e o comportamento de risco para transtornos alimentares. Das que apresentavam maior renda, $37 \%$ apresentaram um comportamento de risco. Entre as de menor renda foi encontrada maior prevalência $(52,1 \%)$, sendo de baixo risco. No BSQ, foi observado que das alunas que possuíam uma menor renda, 83,3\% foram classificadas com ausência de insatisfação corporal, enquanto $32,5 \%$ das que possuíam uma maior renda foram classificadas com insatisfação da imagem corporal.

Boa parte das graduandas residiam com os pais, sendo em sua maioria $(n=66)$ e $21,2 \%$ apresentaram escore positivo para comportamento alimentar de risco. Entre as que moravam com parentes, 57,1\% tiveram EAT positivo para alto risco. Ademais, das 8 estudantes que eram casadas, $5(62,5 \%)$ apresentaram comportamento de risco.

Com relação ao $\mathrm{BSQ}, 78$ alunas que realizavam a maioria das refeições em casa, $61(78,2 \%)$ delas foram classificadas com ausência de insatisfação com a imagem corporal e 21,9\% apresentaram insatisfação. Entretanto, $66,7 \%$ das que faziam as refeições em restaurantes apresentaram algum grau de distorção na imagem corporal. Com relação ao estado civil, 56 estudantes que referiram o estado civil solteira, 76,8\% não apresentaram alguma distorção com a imagem corporal, porém $23,2 \%$ foram classificadas com insatisfação com a imagem corporal.

Os dados relacionados à associação entre idade, IMC atual e desejado e satisfação com a imagem corporal em relação ao comportamento de risco para os transtornos alimentares estão descritos na Tabela 1. Verificou-se que um total de 75 (84,3\%) estudantes demonstraram insatisfação com o seu peso e $26,7 \%$ dessas apresentaram EAT positivo.

Constatou-se associação estatisticamente significativa entre todas as variáveis da Tabela 2. Das que praticavam exercício físico, 36,4\% obtiveram um escore positivo e $15,9 \%$ das que não praticavam apresentaram risco de desenvolver transtornos alimentares. Foi possível observar que $39,3 \%$ realizavam exercício físico com a frequência de 5 a 7 vezes semanais e apresentaram comportamento de risco positivo. Com relação à modalidade, das 44 estudantes que não praticavam alguma 
Tabela 1. Característica das estudantes e sua associação com o comportamento de risco para transtornos alimentares, segundo o EAT-26, João Pessoa - PB, 2019

\begin{tabular}{|c|c|c|c|c|c|c|c|c|c|c|}
\hline \multicolumn{2}{|c|}{ Perfil das Estudantes } & \multicolumn{2}{|c|}{$\begin{array}{c}\text { Fora } \\
\text { de risco }\end{array}$} & \multicolumn{2}{|c|}{$\begin{array}{c}\text { Baixo } \\
\text { risco } \\
\end{array}$} & \multicolumn{2}{|c|}{ Risco } & \multicolumn{2}{|c|}{ Total } & \multirow[t]{2}{*}{$\begin{array}{c}\mathrm{p}- \\
\text { valor* }\end{array}$} \\
\hline & & $\mathrm{n}$ & $\%$ & $\mathrm{n}$ & $\%$ & $n$ & & $\mathrm{n}$ & & \\
\hline \multirow[t]{2}{*}{ Idade em anos } & $17-20$ & 13 & 24,5 & 22 & 41,5 & 18 & 34 & 53 & 100,0 & \\
\hline & $21-40$ & 18 & 39,1 & 19 & 41,3 & 09 & 19,6 & 46 & 100,0 & 0,170 \\
\hline Total & & 31 & 31,3 & 41 & 41,4 & 27 & 27,3 & 99 & 100,0 & \\
\hline \multirow{3}{*}{$\begin{array}{l}\text { Classificação do } \\
\text { IMC atual }\end{array}$} & Baixo peso & 04 & 28,6 & 06 & 42,9 & 04 & 28,6 & 14 & 100,0 & \\
\hline & Eutrofia & 18 & 27,3 & 31 & 47,0 & 17 & 25,8 & 66 & 100,0 & 0,266 \\
\hline & $\begin{array}{c}\text { Excesso de } \\
\text { peso }\end{array}$ & 09 & 56,3 & 04 & 25,0 & 03 & 18,8 & 16 & 100,0 & \\
\hline Total $^{\bullet}$ & & 31 & 32,3 & 41 & 42,7 & 24 & 25,0 & $96^{\bullet}$ & 100,0 & \\
\hline \multirow{3}{*}{$\begin{array}{l}\text { Classificação do } \\
\text { IMC desejado }\end{array}$} & Baixo peso & 03 & 25,0 & 07 & 58,3 & 02 & 16,7 & 12 & 100,0 & \\
\hline & Eutrofia & 24 & 34,8 & 25 & 36,2 & 20 & 29,0 & 69 & 100,0 & 0,373 \\
\hline & $\begin{array}{c}\text { Excesso de } \\
\text { peso }\end{array}$ & - & - & 02 & 50,0 & 02 & 50,0 & 04 & 100,0 & \\
\hline Total $^{\bullet}$ & & 27 & 31,8 & 34 & 40,0 & 24 & 28,2 & $85^{\circ}$ & 100,0 & \\
\hline \multirow{2}{*}{$\begin{array}{l}\text { Satisfação com o } \\
\text { peso }\end{array}$} & Insatisfeita & 24 & 32,0 & 31 & 41,3 & 20 & 26,7 & 75 & 100,0 & 0,967 \\
\hline & Satisfeita & 04 & 28,6 & 06 & 42,9 & 04 & 28,6 & 14 & 100,0 & \\
\hline Total $^{\bullet}$ & & 28 & 31,5 & 37 & 41,6 & 24 & 27,0 & $89^{\circ}$ & 100,0 & \\
\hline
\end{tabular}

Tabela 2. Relação da participação das estudantes em exercícios físicos com comportamento de risco para transtornos alimentares, segundo o EAT-26, João Pessoa - PB, 2019

\begin{tabular}{|c|c|c|c|c|c|c|c|c|c|c|}
\hline \multicolumn{2}{|c|}{ Exercício físico } & \multicolumn{2}{|c|}{$\begin{array}{l}\text { Fora de } \\
\text { risco }\end{array}$} & \multicolumn{2}{|c|}{$\begin{array}{l}\text { Baixo } \\
\text { risco }\end{array}$} & \multicolumn{2}{|c|}{ Risco } & \multicolumn{2}{|c|}{ Total } & \multirow[t]{2}{*}{$\begin{array}{c}\mathrm{p}- \\
\text { valor* }^{*}\end{array}$} \\
\hline & & $n$ & $\%$ & $\mathrm{n}$ & $\%$ & $\mathrm{n}$ & $\%$ & $\mathrm{n}$ & $\%$ & \\
\hline \multirow[t]{3}{*}{ Exercício Físico } & Não & 20 & 45,5 & 17 & 38,6 & 07 & 15,9 & 44 & 100,0 & \\
\hline & Sim & 11 & 20,0 & 24 & 43,6 & 20 & 36,4 & 55 & 100,0 & 0,011 \\
\hline & & 31 & 31,3 & 41 & 41,4 & 27 & 27,3 & 99 & 100,0 & \\
\hline \multirow[t]{2}{*}{ Frequência } & 0 & 20 & 45,5 & 17 & 38,6 & 07 & 15,9 & 44 & 100,0 & \\
\hline & $2-4 x$ & 07 & 25,9 & 11 & 40,7 & 09 & 33,3 & 27 & 100,0 & 0,043 \\
\hline \multirow{2}{*}{ Total } & $5-7 x$ & 04 & 14,3 & 13 & 46,4 & 11 & 39,3 & 28 & 100,0 & \\
\hline & & 31 & 31,3 & 41 & 41,4 & 27 & 27,3 & 99 & 100,0 & \\
\hline \multirow{2}{*}{$\begin{array}{l}\text { Tempo em } \\
\text { minutos }\end{array}$} & 0 & 20 & 45,5 & 17 & 38,6 & 07 & 15,9 & 44 & 100,0 & \\
\hline & $10-50$ & 11 & 25,6 & 18 & 41,9 & 14 & 32,6 & 43 & 100,0 & 0,017 \\
\hline \multirow{2}{*}{ Total } & $60-240$ & - & - & 06 & 50,0 & 06 & 50,0 & 12 & 100,0 & \\
\hline & & 31 & 31,3 & 41 & 41,4 & 27 & 27,3 & 99 & 100,0 & \\
\hline \multirow[t]{2}{*}{ Modalidade } & Nenhuma & 20 & 45,5 & 17 & 38,6 & 07 & 15,9 & 44 & 100,0 & \\
\hline & Uma & 11 & 25,6 & 21 & 48,8 & 11 & 25,6 & 43 & 100,0 & 0,000 \\
\hline \multirow[t]{2}{*}{ Total } & $\begin{array}{c}\text { Duas ou } \\
\text { mais }\end{array}$ & - & - & 03 & 25,0 & 09 & 75,0 & 12 & 100,0 & \\
\hline & & 31 & 31,3 & 41 & 41,4 & 27 & 27,3 & 99 & 100,0 & \\
\hline
\end{tabular}

*Teste Qui-quadrado de Pearson. Fonte: dados da pesquisa, 2018. 
Tabela 3. Características das estudantes e sua associação com a insatisfação da imagem corporal, segundo o BSQ-34, João Pessoa - PB, 2019

\begin{tabular}{|c|c|c|c|c|c|c|c|c|c|}
\hline \multirow{2}{*}{\multicolumn{2}{|c|}{ Perfil das estudantes }} & Ausência & Leve & Moderada & \multicolumn{2}{|c|}{ Grave } & \multicolumn{2}{|c|}{ Total } & \multirow[t]{2}{*}{$p$-valor* } \\
\hline & & n $\%$ & $\begin{array}{ll}\mathrm{n} & \%\end{array}$ & $\mathrm{n} \quad \%$ & $\mathrm{n}$ & & $\mathrm{n}$ & $\%$ & \\
\hline \multirow[t]{2}{*}{ Idade em anos } & $17-20$ & $35 \quad 66,0$ & 17,0 & 13,2 & 023 & 3,8 & 53 & 100,0 & \\
\hline & $21-40$ & 4189,1 & 8,7 & 2,2 & & - & 46 & 100,0 & 0,038 \\
\hline Total & & $76 \quad 76,8$ & $13 \quad 13,1$ & 8,1 & 022 & 2,0 & 99 & 100,0 & \\
\hline Classificação & Baixo peso & $13 \quad 92,9$ & - & 7,1 & - & - & 14 & 100,0 & \\
\hline \multirow[t]{2}{*}{ do IMC atual } & Eutrofia & 5177,3 & 12,1 & 7,6 & 023 & 3,0 & 66 & 100,0 & \\
\hline & $\begin{array}{l}\text { Excesso de } \\
\text { peso }\end{array}$ & 1168,8 & 18,8 & 12,5 & - & - & 16 & 100,0 & 0,642 \\
\hline Total ${ }^{\bullet}$ & & $75 \quad 78,1$ & 11,5 & $\begin{array}{ll}08 & 8,3\end{array}$ & 022 & 2,1 & $96^{\circ}$ & 100,0 & \\
\hline Classificação & Baixo peso & 75,0 & 216,7 & 8,3 & - & - & 12 & $\overline{100,0}$ & \\
\hline do IMC & Eutrofia & 76,8 & 811,6 & 8,7 & 02 & 2,9 & 69 & 100,0 & \\
\hline desejado & $\begin{array}{c}\text { Excesso de } \\
\text { peso }\end{array}$ & 25,0 & 250,0 & $01 \quad 25,0$ & - & - & 04 & 100,0 & 0,327 \\
\hline Total ${ }^{\bullet}$ & & 74,1 & 1214,1 & 9,4 & 2 & 2,4 & $85^{\circ}$ & 100,0 & \\
\hline Satisfação com & Insatisfeita & 76,0 & 912,0 & 9,3 & 02 & 2,7 & 75 & 100,0 & \\
\hline o peso & Satisfeita & 78,6 & 214,3 & 7,1 & - & - & 14 & 100,0 & 0,920 \\
\hline Total $^{\bullet}$ & & 76,4 & 1112,4 & 9,0 & & 2,2 & $89^{\circ}$ & 100,0 & \\
\hline
\end{tabular}

\footnotetext{
*Teste Qui-quadrado de Pearson, •alguns dados foram perdidos. Fonte: dados da pesquisa, 2018.
}

modalidade, $7(15,9 \%)$ tiveram escore $\geq 21$, bem como, das estudantes que praticavam de dois ou mais exercícios nenhuma se encontrava fora de risco e das 12 que praticavam de dois ou mais exercícios, $75,0 \%$ apresentaram comportamento de risco.

Os dados relacionados à associação entre a idade, o IMC atual e desejado e a satisfação com o peso em relação a insatisfação com a imagem corporal estão descritos na Tabela 3. Constatou-se associação estatística significativa entre a variável idade, pois das estudantes que apresentaram uma faixa etária de 17 a 20 anos, 34\% delas demonstraram algum grau de insatisfação com a imagem corporal.

Os dados relacionados às variáveis exercício físico, frequência, tempo e a modalidade em relação à associação com a insatisfação da imagem corporal estão descritos na Tabela 4.

A variável tempo foi estatisticamente 
Tabela 4. Relação da participação das estudantes em exercícios físicos com a insatisfação da imagem corporal, segundo o BSQ-34, João Pessoa - PB, 2019

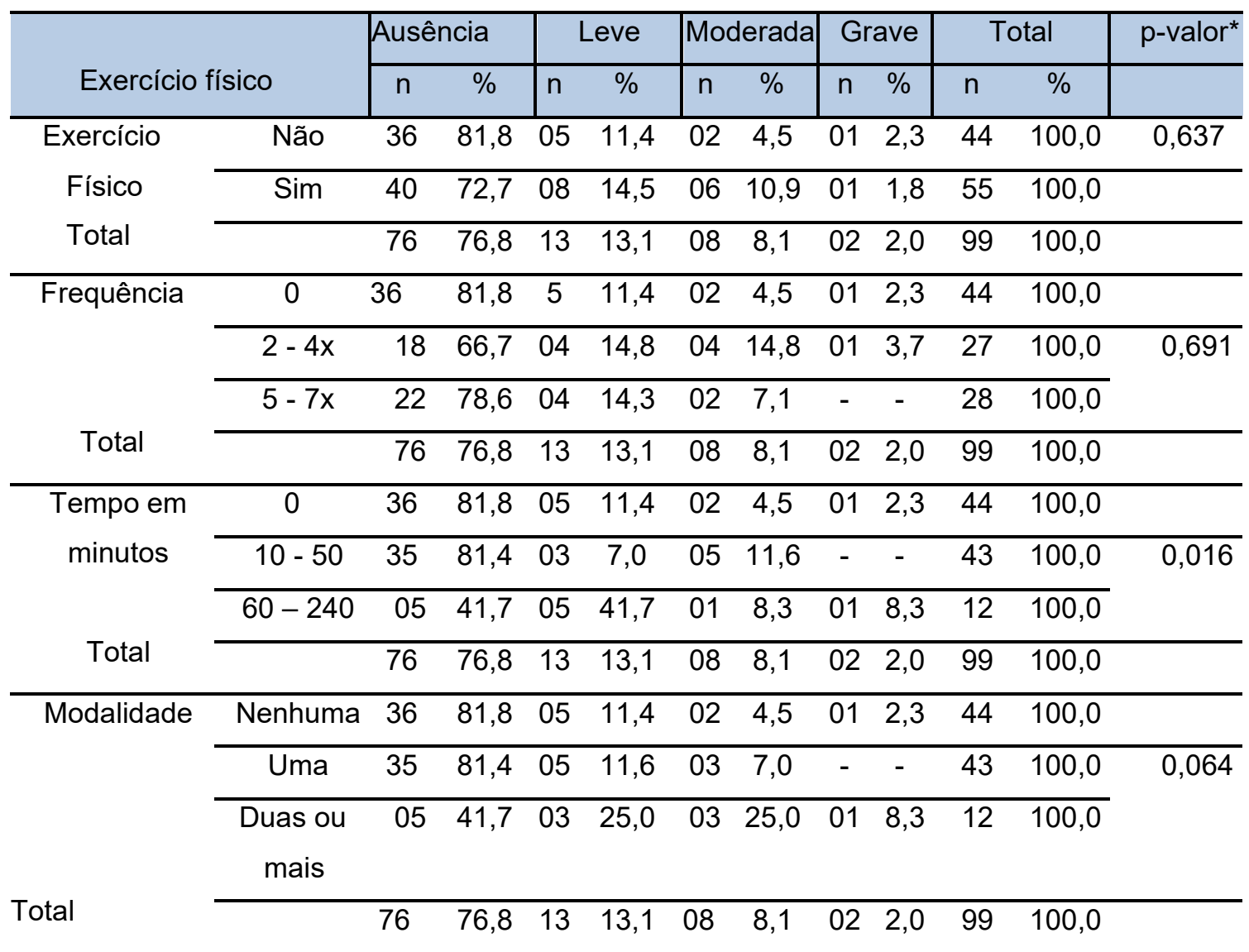

*Teste Qui-quadrado de Pearson. Fonte: dados da pesquisa, 2018.

Tabela 5. Relação entre comportamento de risco para transtornos alimentares e distúrbios de imagem corporal, João Pessoa - PB, 2019

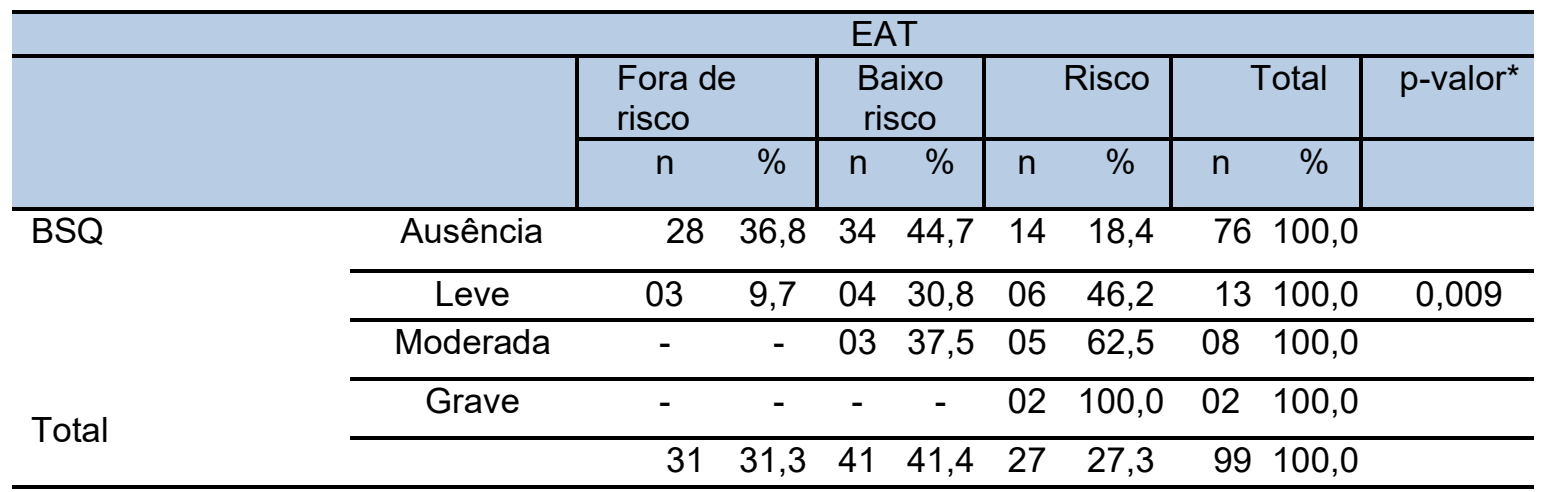

*Teste Qui-quadrado de Pearson. Fonte: dados da pesquisa, 2018. 
significativa, observou-se que 43 estudantes praticavam de 10 a 50 minutos de exercício, sendo que um total de $18,6 \%$ foram classificadas com insatisfação com a imagem corporal.

Conforme os dados expostos na Tabela 5, ocorreu associação estatisticamente significativa entre o EAT com o BSQ. Sendo, portanto, a insatisfação com a imagem corporal relacionada com a presença de atitudes alimentares de risco para o desenvolvimento de transtornos alimentares $(p=0,009)$.

\section{DISCUSSÃO}

O comportamento de risco para transtornos alimentares e a insatisfação com a imagem corporal foram avaliados nesse estudo, no qual foi observada a presença de atitudes alimentares inadequadas, bem como, as de distúrbios de imagem corporal. Constatou-se uma relação entre o comportamento de risco para transtornos alimentares com distúrbios de imagem corporal, assim como, uma associação com algumas variáveis, tais como, renda familiar, idade, prática de exercício físico, frequência, tempo e modalidade do exercício.

A presença de comportamento de risco para transtornos alimentares foi de $27,3 \%$, segundo a classificação do EAT para alto risco de desenvolver transtornos alimentares, resultado ligeiramente acima quando comparado a outros estudos nacionais com universitárias do curso de nutrição. Em estudo recente com estudantes da área de saúde da Universidade Estadual do Ceará (UECE), as universitárias de nutrição apresentaram uma porcentagem de $21 \%$ com EAT positivo ${ }^{15}$. Em outro estudo, também, com estudantes de nutrição da
Universidade Federal de Santa Catarina foi encontrado um percentual de $25,4 \%$, com fator de risco para desenvolvimento de transtorno alimentar, quando comparadas a alunas de outros cursos $(18,7 \%)^{16} \mathrm{e}$, em outro trabalho, também com estudantes de nutrição, foi encontrado um percentual de $21,7 \%$, em que as estudantes apresentaram risco de desenvolverem transtornos alimentares ${ }^{17}$.

É importante ressaltar que, quando o percentual encontrado for maior que $20 \%$ em estudantes com escore EAT positivo, ele é considerado preocupante, no qual evidencia a importância da investigação desses sintomas nas populações que apresentam risco ${ }^{15}$.

Quanto ao estado nutricional, $68,8 \%$ apresentaram um estado nutricional adequado. Valores similares foram encontrados em um estudo com estudantes de nutrição da Cidade de Teresina, da Universidade Federal do Piauí (UFPI), em que a maioria da população estudada apresentou eutrofia, sendo $77,8 \%$ nas estudantes concluintes ${ }^{18}$. Universitárias eutróficas e insatisfeitas com a sua imagem corporal demonstram uma preocupação excessiva com a estética do corpo ${ }^{17}$. Contudo, conforme a associação entre o EAT-26 positivo com o IMC, foi visto que $88,5 \%$ das eutróficas apresentaram EAT positivo, sendo 11,5\% obesas e 2,4\% possuíam o IMC adequado. Analisando os valores do IMC informado e o desejado, pode existir uma forte influência para o limite inferior da normalidade, o que sugere um desejo de adequação ao corpo ideal na atualidade 2 .

Com relação à renda, 48 (51,1\%) alunas possuíam a renda de $R \$ 340,00$ a $R \$$ $3.000,00$. Em outro estudo com estudantes de 
nutrição e administração, em uma instituição de nível superior em Montes Claros-MG, foi observado maior frequência de estudantes com renda familiar na faixa de 2 a 4 salários mínimos, entre os estudantes de nutrição $(68,7 \%)$ e administração $(66,4 \%)$, renda essa que variava de $\mathrm{R} \$ 1.244,00$ a $\mathrm{R} \$ 4.488,00^{19}$. Em uma pesquisa com estudantes do curso da área de saúde de uma universidade pública no interior de Minas Gerais, 51,6\% da amostra possuía renda familiar de 2 até 10 salários $^{20}$. Nesse estudo, a renda apresentou associação com o comportamento de risco. Entre as de menor faixa de renda, constatou-se um percentual significativo de prevalência segundo a classificação de baixo risco $(52,1 \%)$.

Com relação à prática de atividade física, frequência, tempo e modalidade, nesse estudo foi constatado que existiu uma associação com o desenvolvimento de transtornos alimentares. Portanto, $55,6 \%$ acadêmicas praticavam exercícios e $44,4 \%$ não praticavam, porém, $36,4 \%$ das que praticavam exercício físico tiveram EAT positivo. Ademais, 39,3\% das que praticavam de 5 a 7 vezes por semana apresentaram comportamento de risco, quando comparadas com aquelas que realizavam de duas a quatro vezes por semana $(33,3 \%)$ e das que não faziam algum exercício (15,9\%). Resultados esses que se assemelham a outro estudo com alunas de nutrição de uma faculdade particular do Centro do Estado do Rio Grande do Sul, onde se constatou que $37 \%$ das estudantes que mais referiram realizar atividade física, sendo de quatro vezes por semana ou mais, apresentaram mais sintomas associados com transtorno alimentar, quando comparadas com as que realizam até três vezes na semana $(24,6 \%)$ e com as que não realizam atividade física $(21,3 \%)^{21}$. Ainda nesse mesmo estudo, observou-se que, na análise da prática de atividade física, $57 \%$ das estudantes EAT positivo e $47 \%$ com EAT negativo realizavam algum tipo de atividade física durante a semana ${ }^{22}$.

Não foram encontrados estudos nacionais que verificaram a quantificação de modalidades com relação a exercício físico em universitárias, apesar de ter sido constatado associação significativa com os hábitos alimentares inadequados, conforme o EAT positivo, em que $75 \%$ das estudantes que praticavam de duas ou mais modalidades apresentavam comportamento de risco. Dessas modalidades, $36,4 \%$ praticavam musculação, $11 \%$ praticavam musculação e outra modalidade e $8 \%$ praticavam outras modalidades como ballet, pilates, etc. Conforme o estudo com praticantes de atividade física foi avaliado a relação do estado nutricional com a percepção de imagem corporal atual e idealizada. Observou-se que as mulheres possuíam uma imagem corporal distorcida, além de relatos sobre o sentimento de insatisfação com a própria imagem corporal22.

Com relação a variável tempo, 50\% daquelas que tinham como duração o exercício de 60 a 240 minutos apresentaram comportamento alimentar de risco para desenvolvimento de transtornos alimentares. No estudo com frequentadores de uma academia de ginástica ${ }^{23}, 42,5 \%$ dos que praticavam de 60 a 90 minutos apresentaram EAT positivo, quando comparado com aqueles que praticavam até 60 minutos $(31,5 \%)$. Quanto ao $B S Q, 16,6 \%$ que praticavam exercício, com a 
duração de 60 e 240 minutos, apresentaram insatisfação moderada e grave. No estudo com estudantes de Educação Física de uma universidade pública do Rio de Janeiro ${ }^{24}$, verificou-se uma prevalência de insatisfação corporal de $14,6 \%$ entre aqueles que praticavam exercício de 60 a 90 minutos, segundo a classificação de moderada ou grave insatisfação conforme o BSQ.

De acordo com a análise do BSQ, $76,8 \%$ das estudantes foram classificadas com ausência de insatisfação da imagem corporal e $23,2 \%$ com presença de insatisfação. Constatou-se que desses $23,2 \%, 10,1 \%$ das universitárias apresentaram de moderada a grave insatisfação de acordo com a classificação do BSQ. Essa prevalência é semelhante à insatisfação encontrada em outro estudo com estudantes de nutrição da Universidade Federal do Rio Grande do Sul (UFRGS), na Cidade de Porto Alegre, sendo um percentual de $13,5 \%{ }^{25}$ e com universitárias do Curso de Nutrição da Universidade Federal de Ouro Preto (UFOP), em que foi encontrado um total de $13,7 \%{ }^{17}$. Sobretudo, outros estudos encontraram percentuais maiores como em um estudo, no Rio de Janeiro, sendo de 18,6\% ${ }^{2}$.

No presente estudo foi observada a associação entre a insatisfação com a imagem corporal e o comportamento de risco para transtornos alimentares $(p<0,009)$, bem como, o estudo realizado em uma instituição de educação superior da Região Oeste do Paraná, com estudantes de educação física. Verificou-se a associação das variáveis com transtorno alimentar, identificando associação significativa com a imagem corporal. Os universitários com presença de distorção da imagem corporal apresentaram prevalência de transtorno alimentar 5,6 vezes maior que aqueles sem distorção da imagem corporal, mesmo com ajustes por sexo e estado nutricional $^{26}$. Da mesma forma, em outra pesquisa ${ }^{19}$ ocorreu associação estatística entre a percepção da imagem corporal e a anorexia nervosa $(p=0,000)$ e a bulimia nervosa $(p=0,000)$, conforme a escala de silhuetas ${ }^{20}$, além de outro estudo em que foi encontrada associação estatisticamente significativa $(p=0,000)$ entre a imagem corporal e o comportamento alimentar inadequado ${ }^{25}$. Da mesma forma, a chance de presença de comportamento bulímico nos universitários em Florianópolis, que foi 19,9 vezes maior naqueles com distorção da imagem corporal27.

Diante do exposto, é possível perceber que os resultados desse estudo são bem preocupantes, visto que, existe um grande número de participantes do estudo com insatisfação com o peso, evidenciando um comportamento de risco para transtornos alimentares e distúrbios de imagem corporal. Esses números são expressivos, visto que serão futuras profissionais da área de saúde, das quais serão promotoras de saúde e, não obstante a isso, possuem contato constante com o alimento e informações nutricionais acerca dos mesmos. É fato que a cobrança da sociedade perante esses futuros profissionais é tamanha, que faz com que exista uma maior preocupação quanto à aparência física. Esses fatores podem predispor a maior suscetibilidade de desenvolver algum tipo de distúrbio alimentar e corporal.

No entanto, é imprescindível que a instituição de ensino possa estabelecer um 
olhar diferenciado para os acadêmicos, sendo necessário que haja a promoção de saúde, por meio de programas que estimulem os universitários a ter uma melhor qualidade de vida, pois em um quadro de transtornos alimentares é importante que exista uma maior atenção no sentido de esclarecer e discutir sobre o tema, a fim de prevenir a prática de atitudes alimentares consideradas anormais entre os estudantes de uma forma geral.

Por fim, os dados apresentados nesse estudo demonstram a importância de ser avaliado o comportamento alimentar, os hábitos de vida, a prática de exercício físico e a imagem corporal em universitárias, principalmente aquelas da área da saúde, em especial, as de nutrição, visto que são consideradas um grupo de risco, uma vez que as mesmas irão lidar com pessoas que poderão possuir algum tipo de transtorno alimentar e distúrbio de imagem corporal, para que não se perpetue a conduta de práticas que ocasionem o início ou a manutenção de um distúrbio alimentar.

O presente estudo apresenta algumas limitações que incluem o fato do estudo ser do tipo transversal, pois não permite um acompanhamento contínuo, mas apenas o estabelecimento de uma relação temporal entre as variáveis abordadas, além do uso de questionários, os quais dependem mais do entendimento de quem está respondendo, podendo existir interpretações equivocadas. Quanto ao uso dos questionários, por terem sido usados instrumentos validados, minimiza esse efeito da compreensão.

\section{CONCLUSÃO}

Os resultados encontrados nesse estudo demonstram que existe uma hipervalorização de corpo ideal, excepcionalmente o magro, o que prevalece de acordo com os resultados constatados, pois ocorreu uma grande insatisfação com o peso corporal. Além disso, identificou-se uma alta prevalência com relação ao comportamento alimentar de risco para transtornos alimentares e presença de distúrbios de imagem corporal, além da insatisfação com a imagem corporal pelo desejo de perder peso. Foram encontradas associações entre a idade e o tempo do exercício físico com a insatisfação da imagem corporal e, também, associações entre o perfil socioeconômico, prática de exercício físico, o tempo do exercício, a modalidade e a frequência com o comportamento de risco para transtornos alimentares. Esses dados são de extrema relevância, visto que entre a relação do IMC atual com o desejado foi observado o desejo explícito de perda de peso, até mesmo entre as estudantes que se encontravam com o peso adequado para a sua estatura. Esses resultados apontam para a necessidade de uma atenção especial a esse grupo de risco, pois o papel, como futuras profissionais, implica diretamente em como será a abordagem diante de um quadro clínico similar para que não haja a prática inadequada de controle de peso e informações errôneas. 


\section{REFERÊNCIAS}

1. Alvarenga MS, Philippi, ST, Lourenço BH, Sato PM, Scagliusi, FB. Insatisfação com a imagem corporal em universitárias brasileiras. J Bras Psiquiatr. 2010; 59(1):4451.

2. Bosi MLM, Luiz RR, Morgado CMC, Costa Mara LS, Carvalho RJ. Autopercepção da imagem corporal entre estudantes de nutrição: um estudo no município do Rio de Janeiro. J Brasi Psiquitr. 2006; 55(2):108-113.

3. Petroski EL, Pelegrini A, Glaner MF. Motivos e prevalência de insatisfação com a imagem corporal em adolescentes. Cien Saude Colet. 2012; 17(4):1071-1077.

4. Alves TC, Santana MLP, Silva RCR, Pinto EJ, Assis AMO. Fatores associados a sintomas de transtornos alimentares entre escolares da rede pública da cidade do Salvador, Bahia. J Bras Psiquiatr. 2012; 61(2):55-63.

5. Alvarenga MS, Scagliusi FB, Philippi ST. Comportamento de risco para transtorno alimentar em universitárias brasileiras. Rev Psiquiatr Clín. 2011; 38(1):3-7.

6. Souto S, Ferro-bucher JSN. Práticas indiscriminadas de dietas de emagrecimento e o desenvolvimento de transtornos alimentares. Rev Nutr. 2006; 19(6):693-704.

7. Castro PS, Brandão ER. Tomando a anorexia nervosa como objeto de estudo socioantropológico: aproximação com os sujeitos da pesquisa. DEMETRA. 2014; 9(1):3-22.

8. Alvarenga MS, Scagliusi FB. Tratamento nutricional da bulimia nervosa. Rev Nutr. 2010; 23(5):907-918.

9. Timerman F, Scagliusi FB, Cordás TA. Acompanhamento da evolução dos distúrbios de imagem corporal em pacientes com bulimia nervosa, ao longo do tratamento multiprofissional. Rev Psiquiatr Clín. 2010; 37(3):113-117.

10. Nunes MA, Bagatini LF, Abuchaim AL, Kunz A, Ramos D, Silva JA, et al. Distúrbios da conduta alimentar: considerações sobre o teste de atitudes alimentares (EAT-26). Rev ABP-APAL. 1994; 16(1):7-10.

11. Di Pietro M, Silveira DX. Internal validity, dimensionality and performance of the Body Shape Questionnaire in a group of Brazilian college students. Rev Brasi Psiquiatr. 2009; 31(1):4-21

12. Garner DM, Garfinkel PE. The Eating Attitudes Test: an index of the symptom of anorexia nervosa. Psychol Med.1979;9(2):273-279.

13. Magalhães VC, Mendonça GAS. Transtornos alimentares em universitárias: estudo de confiabilidade da versão brasileira de questionários autopreenchíveis. Rev Bras Epidemiol. 2005; 8(3):236-245.
14. Cooper PJ, Taylor J, Cooper Z, Fairburn G. The development and validation of the body shape questionnaire. Int J Eat Disord., 1987; 6(4):485-494.

15. Sampaio HAC, Silva IA, Parente NA, Carioca AAF Ambiente familiar e risco de transtorno alimentar entre universitários da área de saúde. DEMETRA. 2019; 14(33308):1-15.

16. Fiates GMR, Salles RK. Fatores de risco para o desenvolvimento de distúrbios alimentares: um estudo em universitárias. Rev Nutr. 2001; 14(Supl 0):3-6.

17. Silva JD, Silva ABJ, Oliveira AVK, Nemer ASA. Influência do estado nutricional no risco para transtornos alimentares em estudantes de nutrição. Ciênci. Saúde Coletiva. 2012; 17(12):3399-3406.

18. Lopes MAM, Paiva AA, Lima SMT, Cruz KJC, Rodrigues GP, Carvalho CMRG. Percepção da imagem corporal e estado nutricional em acadêmicas de nutrição de uma universidade pública. DEMETRA. 2017; 12(1):193-206.

19. Moreira DE, Pinheiro MC, Carreiro DL, Coutinho LTM Almeida KTCL, Santos CA, Coutinho WLM, et al. Transtornos alimentares, percepção da imagem corporal e estado nutricional: estudo comparativo entre estudantes de nutrição e de administração. RASBRAN. 2017; $8(1): 18-25$

20. Costa DG, Carleto CT, Santos VS, Haas VJ, Gonçalves RMDA, Pedrosa LAK. Qualidade de vida e atitudes alimentares de graduandos da área da saúde. Rev Bras Enferm. 2018; 71(Suppl 4):1739-1746.

21. Kirsten VR, Fratton F, Porta NBD. Transtornos alimentares em alunas de nutrição do Rio Grande do Sul. Rev Nutri. 2009; 22(2):219-227.

22. Cosio RBZ, Romeiro AT, Rossi L. Avaliação da percepção da imagem corporal em uma academia do município de São Paulo. Rev Digital. 2008; 13(123):6-7

23. Weis A. Transtorno alimentar e variáveis comportamentais relacionadas ao exercício físico em academia de ginástica. [Dissertação de Mestrado] Curitiba: Universidade Federal do Paraná; 2013. 74p.

24. Marques RS, Oliveira AP, Assis MR. Prevalência de insatisfação com a imagem corporal entre estudantes de educação física. Corpus et Scientia. 2013; 9(1):65-78.

25. Garcia CA, Castro TG, Soares RM. Comportamento alimentar e imagem corporal entre estudantes de Nutrição de uma universidade pública de Porto Alegre-RS. Revista HCPA, 2010; 30(3):219-224. 
26. Legnani RFS, Legnani E, Pereira EF, Gasparotto GS, Vieira LF, Campos W. Transtornos alimentares e imagem corporal em acadêmicos de Educação Física. Motriz. 2012; 18(1):84-91.

27. Cenci M, Peres KG, Vasconcelos FAG. Prevalência comportamento bulímico e fatores associados em universitárias. Rev Psiquiatri Clín. 2009; 36(3):83-88.

\section{CORRESPONDÊNCIA}

Michelle Cristine Nascimento de Melo Endereço postal da autora: Avenida Liberdade, 4418 Centro - Bayeux - PB, Brasil, 58306-001.

E-mail: michellecristinemelo@gmail.com 